\title{
Doenças de chinchilas (Chinchilla lanigera) ${ }^{1}$
}

\author{
Ricardo B. Lucena², Paula R. Giaretta3 ${ }^{3}$, Bianca Tessele 3 , Rafael A. Fighera4, \\ Glaucia D. Kommers ${ }^{4}$, Luiz Francisco Irigoyen ${ }^{4}$ e Claudio S.L. Barros ${ }^{4 *}$
}

\begin{abstract}
Lucena R.B., Giaretta P.R., Tessele B., Fighera R.A., Kommers G.D., Irigoyen L.F. \& Barros C.S.L. 2012. [Diseases of chinchilla (Chinchilla lanigera).] Doenças de chinchilas (Chinchilla lanigera). Pesquisa Veterinária Brasileira 32(6):529-535. Departamento de Patologia, Universidade Federal de Santa Maria, 97105-900 Santa Maria, RS, Brazil. E-mail: claudioslbarros@uol.com.br

Diseases of chinchilla were studied through the review of necropsy reports of 202 postmortem examinations carried out in this species from January 1997 to December 2011. One hundred and eighty nine of these necropsies (93.5\%) had a conclusive diagnosis and in $13(6.5 \%)$ a conclusive diagnosis was not reached due either absence of lesions or advanced autolysis. One hundred and sixty two (80\%) of the necropsied chinchilla were females and 37 (18\%) were males and the sex was not recorded in four cases (2\%). Ages of necropsied chinchillas varied from one day to 12-years-old. The encountered diseases were grouped in the following categories: inflammatory diseases, diseases caused by intoxications, diseases caused by physical agents, metabolic diseases, parasitism, degenerative diseases, circulatory disturbances, developmental disorders and "other disorders". Inflammatory diseases were the most prevalent (52 cases [25.7\%]) and included gastritis (10 cases), necrotizing enteritis (6 cases), listeriosis (5 cases), septicemia (5 cases), bacterial bronchopneumonia (4 cases), pyometra (4 cases), diarrhea associated with Proteus sp. ( 3 cases), subcutaneous and lymph node abscesses (2 cases), endometritis ( 2 cases), otitis ( 2 cases), pyelonephritis ( 2 cases), abscesses in hepatic the round ligament (1 cases), fibrinous pneumonia (1 case), interstitial pneumonia (1 case), hepatitis and cholecystitis associated with Salmonella sp. (1 case), pulmonary histiocytosis (1 case), and dermatophytosis by Trichopyton metagrophytes (1 case). The second most prevalent group of diseases was that caused by intoxications $(22,3 \%$ of the cases) including 45 cases of intoxication by salinomycin. Diseases caused by physical agents (21 cases [10.4\%]) included trauma cases caused by other animals ( 8 cases), self mutilation secondary to intramuscular injection (8 cases), rectal prolapsed ( 3 cases) and dystocia (2 cases). Metabolic diseases were represented by 16 cases $(7.9 \%)$ of hepatic lipidosis. Parasitic diseases were represented by 8 cases $(4 \%)$ flea ( 4 cases) lice ( 3 cases) infestations and one case o giardiasis. Degenerative diseases ( 4 cases or $2,5 \%$ ) included two cases of chronic renal failure, one case of scattered hepatocellular necrosis and one case of muscle necrosis of unknown origin. Circulatory disturbances included two cases $(0.99 \%)$ of congestive heart failure. Neoplasms were represented by two cases $(0.99 \%)$ of gastric adenocarcinoma. Developmental disorders included one (0,5\%) case of atresia ani associated with polycystic kidneys and absence of reproductive tract and large intestine. Thirty eight cases (18.8\%) did not fit in any of the above categories and were placed as "other disorders". In this category dental disease was the most commonly (8 cases or 9\%) diagnosed disorder, followed by 14 cases $(6,9 \%)$ of hyperthermia, tow cases of anemia, two cases of fat metaplasia of adrenal cortex and two cases of mucometra.
\end{abstract}

INDEX TERMS: Chinchilla, causes of death, retrospective study, prospective study, epidemiology, pathology.

${ }^{1}$ Recebido em 2 de fevereiro de 2012.

Aceito para publicação em 24 de fevereiro de 2012.

${ }^{2}$ Programa de Pós-Graduação em Medicina Veterinária, área de concentração em Patologia Veterinária, Centro de Ciências Rurais (CCR), Universidade Federal de Santa Maria (UFSM), Camobi, Santa Maria, RS 97105-900, Brasil.

\footnotetext{
${ }^{3}$ Graduação em Medicina Veterinária, Laboratório de Patologia Veterinária (LPV), CCR-UFSM, Santa Maria, RS.

${ }^{4}$ Departamento de Patologia, Centro de Ciências da Saúde, UFSM, Avenida Roraima 1000, Santa Maria, RS. *Autor para correspondência: claudioslbarros@uol.com.br
} 
RESUMO.- As doenças de chinchilas foram estudadas através da avaliação de laudos de necropsia entre janeiro de 1997 e dezembro de 2011. Em 202 chinchilas necropsiadas, $189(93,5 \%)$ tiveram o diagnóstico determinado, e 13 $(6,5 \%)$ tiveram diagnóstico inconclusivo, por ausência de lesões ou autólise acentuada. Dentre as 202 chinchilas computadas, 162 eram fêmeas (80\%), 37 eram machos (18\%), e em quatro chinchilas (2\%) o sexo não foi anotado. As chinchilas tinham entre um dia a 12 anos de idade. As doenças foram agrupadas nas seguintes categorias: doenças inflamatórias, doenças causadas por intoxicações, doenças causadas por agentes físicos, doenças metabólicas, doenças parasitárias, doenças degenerativas, distúrbios circulatórios, neoplasmas, distúrbios do desenvolvimento e "outros distúrbios". As doenças inflamatórias foram as mais prevalentes (52 casos [25,7\%]) e foram representadas por casos de gastrite (10 casos), listeriose (5 casos), septicemia (5 casos), broncopneumonia bacteriana (4 casos), enterite necrosante (4 casos), piometra (4 casos), diarreia com isolamento de Proteus sp. (3 casos), abscessos subcutâneos e em linfonodos ( 2 casos), endometrite ( 2 casos), otite ( 2 casos), pielonefrite ( 2 casos), abscesso do ligamento redondo do fígado ( 1 caso), pneumonia fibrinosa ( 1 caso), pneumonia intersticial (1 caso), hepatite e colecistite com isolamento de Salmonella sp. (1 caso), histiocitose pulmonar (1 caso), miosite linfo-histiocítica (1 caso) e um caso de dermatofitose (Trichopyton metagrophytes). 0 segundo grupo de doenças mais prevalentes foram as intoxicações $(22,3 \%)$, representado por 45 casos de intoxicação por salinomicina. As doenças causadas por agentes físicos (21 casos [10,4\%]) incluíam casos de traumas causados por outros animais (8 casos), automutilação após injeção intramuscular (8 casos), prolapso de reto ( 3 casos) e parto distócico ( 2 casos). A categoria de doenças metabólicas foi representada por 16 casos $(7,9 \%)$ de lipidose hepática. As doenças parasitárias (8 casos [4\%]) consistiram em infestação por pulga (4 casos), piolho ( 3 casos) e giardíase (1 caso). Doenças degenerativas (4 casos [2,5\%]) incluíam insuficiência renal crônica (2 casos), necrose aleatória de hepatócitos (1 caso) e necrose muscular de origem desconhecida (1 caso). Os distúrbios circulatórios incluíram dois casos $(0,99 \%)$ de insuficiência cardíaca congestiva. Neoplasmas foram representados por dois casos $(0,99 \%)$ de adenocarcinoma gástrico. Um caso de atresia ani, associado a ausência do trato reprodutor, intestino grosso e rins policíticos representou a categoria de distúrbios do desenvolvimento $(0,5 \%)$. Algumas doenças não se enquadraram nas categorias acima e foram enquadradas em "outros distúrbios" (38 casos [18,8\%]). Nesta categoria, doenças dentárias foi o distúrbio mais comum, diagnosticado em 9\% (18 de 202) de todas as chinchilas examinadas. Seguido por casos de hipertermia (14 casos), dois casos de anemia, dois casos de metaplasia de células adiposas do córtex da adrenal, e dois casos de mucometra.

TERMOS DE INDEXAÇÃO: Chinchilas, causas de morte, estudo retrospectivo, estudo prospectivo, epidemiologia, patologia.

\section{INTRODUÇÃO}

Chinchilas (Chinchilla lanigera) são mamíferos histricomorfos pertencentes à ordem Rodentia e família Chinchillidae, nativas dos Andes, com distribuição natural restrita ao Chile, Bolívia e Peru (Bidlingmaier 1937). Há duas espécies, Chinchilla brevicaudata originária da Bolívia e Chinchilla lanigera encontrada principalmente no Chile (Boone 1933, Bidlingmaier 1937). A espécie $C$. lanigera foi domesticada e atualmente há milhões de chinchilas criadas em cativeiro por todo o mundo (Boone 1933, Bidlingmaier 1937, Jimenez 1996). Embora as chinchilas tenham sido originalmente domesticadas visando a produção de pele, o que ocorre até hoje, há também, considerável aumento de chinchilas criadas como animais de companhia e de laboratório (Donnelly 2004).

$\mathrm{Na}$ literatura são encontrados poucos os estudos sobre as doenças de chinchilas, na sua maioria são estudos antigos, das décadas de 1950 e 1960, ou são repetições e revisões de literatura (Donnelly 2004). Ultimamente tem-se observado aumento do número de cadáveres de chinchilas remetidos para necropsia no Laboratório de Patologia Veterinária da UFSM (LPV-UFSM). Além de diferentes lesões, casos de gastrite são frequentemente observados, associados ou não a sinais clínicos. A avaliação histológica do estômago em muitas dessas chinchilas revela gastrite associada à atrofia gástrica e diferentes graus de metaplasia intestinal e displasia. Lipidose hepática é outra lesão comumente encontrada em chinchilas na rotina de diagnóstico (dados não publicados).

0 presente estudo tem como objetivo avaliar os aspectos epidemiológicos, sinais clínicos e os achados patológicos das doenças que acometem chinchilas, através do estudo dos casos de necropsia de chinchilas realizadas no LPV-UFSM entre os anos 1997-2011.

\section{MATERIAL E MÉTODOS}

Foram revisados os protocolos de necropsia de chinchilas realizadas no LPV-UFSM, de janeiro de 1997 a dezembro de 2011 (15 anos). Desses, foram computadas informações quanto à idade, ao sexo, os sinais clínicos, as alterações laboratoriais, as lesões macroscópicas e histopatológicas e, o diagnóstico definitivo. Aqueles protocolos de necropsia que não apresentavam um diagnóstico definitivo ou que não continham informações que permitissem concluir por um diagnóstico foram considerados como inconclusivos.

Após a compilação dos diagnósticos, os dados foram agrupados nas seguintes categorias: distúrbios circulatórios, distúrbios do desenvolvimento, doenças causadas por agentes físicos, doenças degenerativas, doenças inflamatórias, doenças parasitárias, doenças metabólicas, doenças causadas por intoxicações e neoplasmas. Os distúrbios que não puderam ser enquadrados em nenhuma dessas categorias foram agrupados sob a denominação de "outros distúrbios". Além da identificação da causa da morte foram computados os achados incidentais de necropsia. Para uma melhor distribuição dos distúrbios quanto à faixa etária, as chinchilas foram divididas em cinco grandes grupos de idade, tendo em vista sua longa longevidade: filhotes em lactação (desde o nascimento até os dois meses de idade que é a idade de desmame adotada pelos criadores); jovens (dos três aos oito meses de idade); adultos jovens (a partir dos nove meses de idade, quando entram em idade reprodutiva, até dois anos); adultos (de dois aos nove anos de idade); e idosos (dez anos de idade ou mais).

\section{RESULTADOS}

Durante o período de 1997-2011 foram necropsiadas 252 chinchilas no LPV-UFSM. Dessas, 202 morreram esponta- 
neamente ou foram submetidas à eutanásia por apresentarem alguma doença. As outras 50 necropsias dizem respeito ao estudo de carcaças de chinchilas saudáveis abatidas para obtenção da pele, desta forma, não foram consideradas no presente trabalho. Dentre as 202 chinchilas computadas, 162 eram fêmeas (80\%), 37 eram machos (18\%), e quatro chinchilas não tiveram o sexo definido (2\%) devido extensa mutilação em recém-nascidos pelas mães, e em um caso de malformação com ausência do trato reprodutor. A idade dos animais variou de um dia a 12 anos de idade. A prevalência das doenças diagnosticadas em chinchilas de acordo com a categoria de doença e idade dos animais, entre os anos de 1997-2011 estão sumarizadas no Quadro 1.

As doenças inflamatórias foram as mais prevalentes, correspondendo a 25,7\% (52 de 202 casos) de todas as chinchilas avaliadas. Dentre as doenças infecciosas prevaleceram os casos de gastrite (10 casos) associado a bactérias espiraladas na mucosa, interpretadas como Helicobacter sp. Gastrite, também, foi observada em outros casos como achados incidentais e não associado com a morte desses animais. Listeriose foi diagnostica em cinco animais, provenientes de três surtos. Septicemia foi diagnosticada em 5 chinchilas. Outras doenças inflamatórias incluíram enterite necrosante ( 6 casos), broncopneumonia bacteriana (4 casos), piometra (4 casos), diarreia com isolamento de Proteus sp. (3 casos), abscessos subcutâneos e linfáticos (2 casos), endometrite (2 casos), otite ( 2 casos), pielonefrite (2 casos), abscesso do ligamento redondo do fígado (1 caso), pneumonia fibrinosa (1 caso), pneumonia intersticial (1 caso), colecistite e hepatite com isolamento de Salmonella sp. (1 caso), histiocitose pulmonar (1 caso), miosite linfo-histiocítica (1 caso) e um caso de dermatofitose (Trichopyton metagrophytes).

As intoxicações também tiveram grande número de casos, devido a necropsia de 45 chinchilas provenientes de importantes surtos de intoxicação por salinomicina, diagnosticados em quatro propriedades que forneceram ração de um mesmo lote contaminado aos seus animais.

As doenças causadas por agentes físicos também tiveram grande representação (21 casos), principalmente devido aos casos de trauma (8 casos) provocado por outras chinchilas (Fig.1) ou animais maiores, como também casos de automutilação (8 casos) (Fig.2) após administração de enrofloxacina via intramuscular. Outros casos associados a lesões traumáticas incluem cinco mortes de chinchilas com constipação intestinal (5 casos), prolapso de reto (3 casos) e parto distócico (Fig.3) (2 casos).

A categoria doenças metabólicas foi representada por protocolos em que constavam lipidose hepática (Fig.4) como principal ou única lesão encontrada (16 casos), porém, degeneração gordurosa do fígado foi um achado incidental frequente em chinchilas que morreram por outras causas, e que passaram por período de restrição alimentar.

Doenças parasitárias foram diagnosticas em oito chinchilas. Quatro delas infestadas por pulgas, três por piolhos e uma com giardíase. Dentre as doenças degenerativas, foram computados casos de insuficiência renal crônica (2 casos), necrose aleatória de hepatócitos de origem não determinada (1 caso) e de necrose muscular (1 caso), também de etiologia desconhecida. Os distúrbios

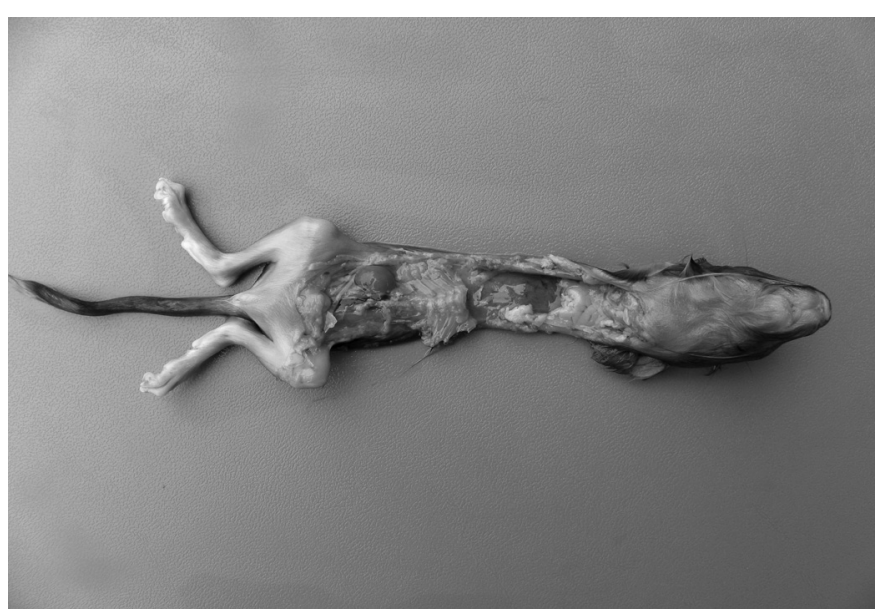

Fig.1. Mutilação de uma chinchila recém-nascida provocada pela mãe algumas horas após o parto. Foram ingeridos os membros torácicos, os músculos abdominais, o esterno e praticamente todas as vísceras da cavidade abdominal e torácica, restando apenas o rim direito e parte do timo.

Quadro 1. Relação entre as categorias de idade e as diferentes categorias de doenças diagnosticadas em chinchilas no Laboratório de Patologia Veterinária da Universidade Federal de Santa Maria entre 1964-2008

\begin{tabular}{|c|c|c|c|c|c|c|c|}
\hline \multirow[t]{2}{*}{ Categorias de doenças } & \multicolumn{6}{|c|}{$\begin{array}{l}\text { Prevalências das categorias de doenças por faixa } \\
\text { etária (número de casos) }\end{array}$} & \multirow{2}{*}{$\begin{array}{c}\text { Total/ \% } \\
\text { dos } 202 \\
\text { casos }\end{array}$} \\
\hline & Filhote & Jovem & $\begin{array}{l}\text { Adulto } \\
\text { jovem }\end{array}$ & Adulto & Idosa & $\begin{array}{l}\text { Idade não } \\
\text { informada }\end{array}$ & \\
\hline Doenças inflamatórias & 6 & 9 & 10 & 19 & 8 & - & $52(25,7 \%)$ \\
\hline Doenças tóxicas & 12 & 4 & 14 & 15 & - & - & $45(22,3 \%)$ \\
\hline Causados por agentes físicos & 6 & 9 & 4 & 2 & - & - & $21(10,4 \%)$ \\
\hline Doenças metabólicas & 2 & 3 & 6 & 3 & 1 & 1 & $16(7,9 \%)$ \\
\hline Doenças parasitárias & 2 & 1 & - & 4 & 1 & - & $8(4,0 \%)$ \\
\hline Doenças degenerativas & - & - & 1 & 2 & 1 & - & $4(1,9 \%)$ \\
\hline Distúrbios circulatórios & - & 1 & - & 1 & - & - & $2(1,0 \%)$ \\
\hline Neoplasmas & - & - & - & 1 & 1 & - & $2(1,0 \%)$ \\
\hline Distúrbios do desenvolvimento & 1 & - & - & - & - & - & $1(0,5 \%)$ \\
\hline Outros distúrbios & - & 4 & 9 & 21 & 2 & 2 & $38(18,8 \%)$ \\
\hline Inconclusivo & 1 & 6 & 3 & 2 & - & 1 & $13(6,4 \%)$ \\
\hline TOTAL & 30 & 37 & 47 & 70 & 14 & 4 & 202 \\
\hline
\end{tabular}




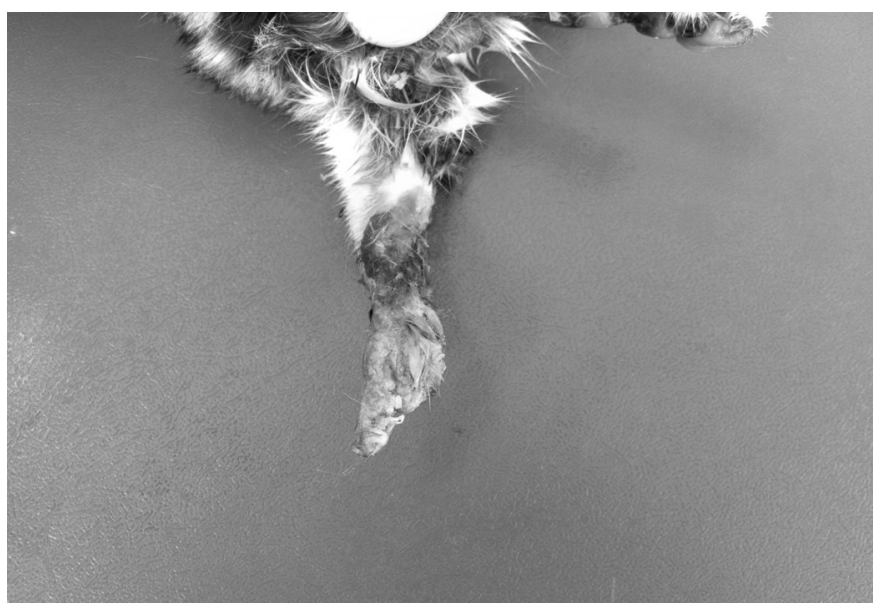

Fig.2. Automutilação de parte do membro pélvico direito de uma chinchila após injeção intramuscular de enrofloxacina. A chinchila decepou parte dos dígitos e da pele dorsal e plantar.

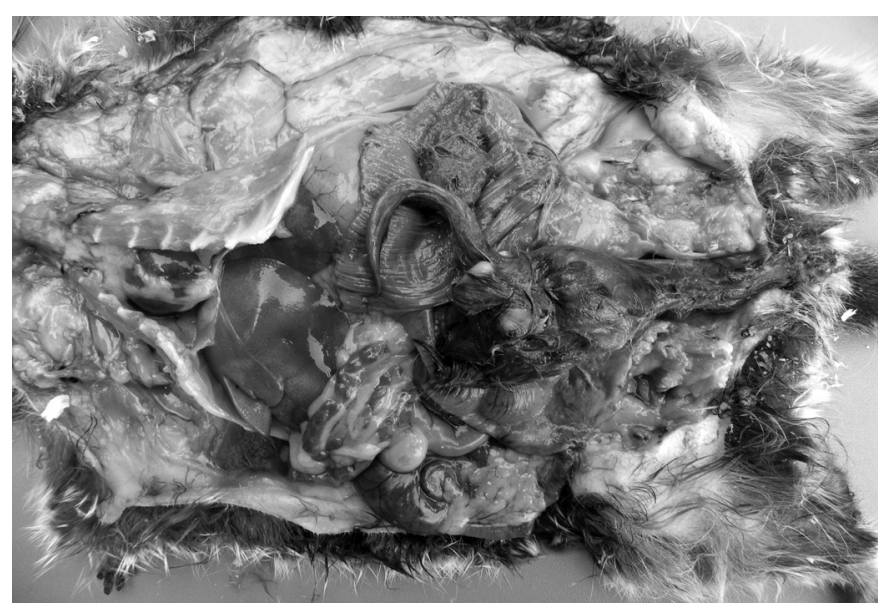

Fig.3. Parto distócico. Um feto grande está mal posicionado, com o dorso voltado para a abertura do canal do parto. 0 fígado está pálido e aumento de volume (lipidose hepática).

circulatórios se limitaram a dois casos de insuficiência cardíaca congestiva. Neoplasmas foram representados por dois casos de adenocarcinoma gástrico. A categoria de distúrbios do desenvolvimento foi representada por um caso de malformações múltiplas em uma chinchila recém-nascida.

Algumas doenças não se enquadraram nas categorias acima e foram enquadradas em "outros distúrbios" (38 casos). Nesta categoria, doenças dentárias foi o distúrbio mais comum, diagnosticado em 9\% (18 de 202) de todas as chinchilas examinadas (Fig.5). Seguido por casos de hipertermia (14 casos), ocorridos principalmente nos meses de verão. Outros casos incluiram anemia ( 2 casos), metaplasia de células adiposas do córtex da adrenal associado a alopecia nas regiões ventrolateral do abdômen e ventral cervical ( 2 casos) e mucometra ( 2 casos).

Em 13 casos não foram observadas lesões macroscópicas ou histológicas, ou o animal estava em avançado grau de autólise, que comprometeu sua avaliação. Esses casos foram computados como diagnóstico inconclusivo.

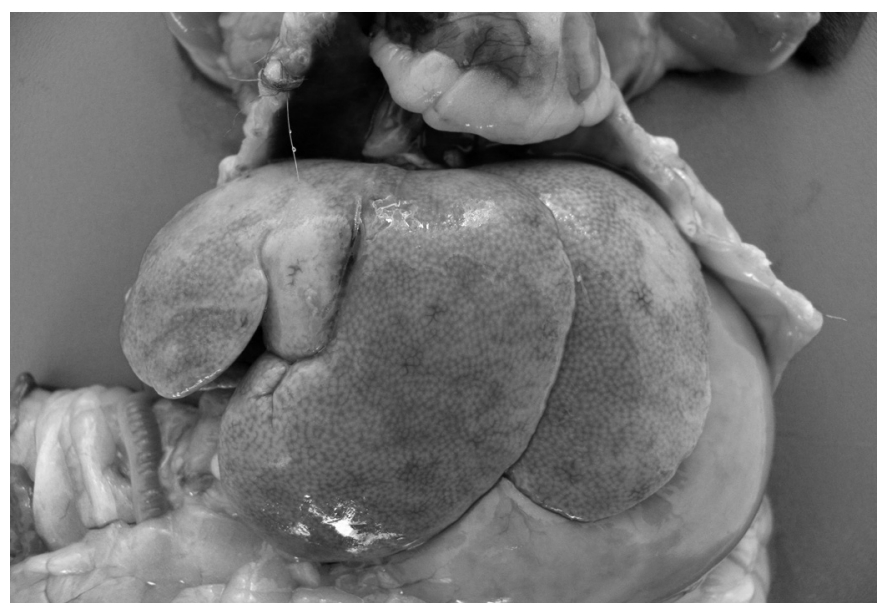

Fig.4. Marcada lipidose hepática em uma chinchila que morreu em decorrência de hipertermia. 0 fígado está pálido, aumentado de volume, com as bordas arredondadas. A lipidose hepática é uma consequência do quadro de anorexia desenvolvido pela chinchila.

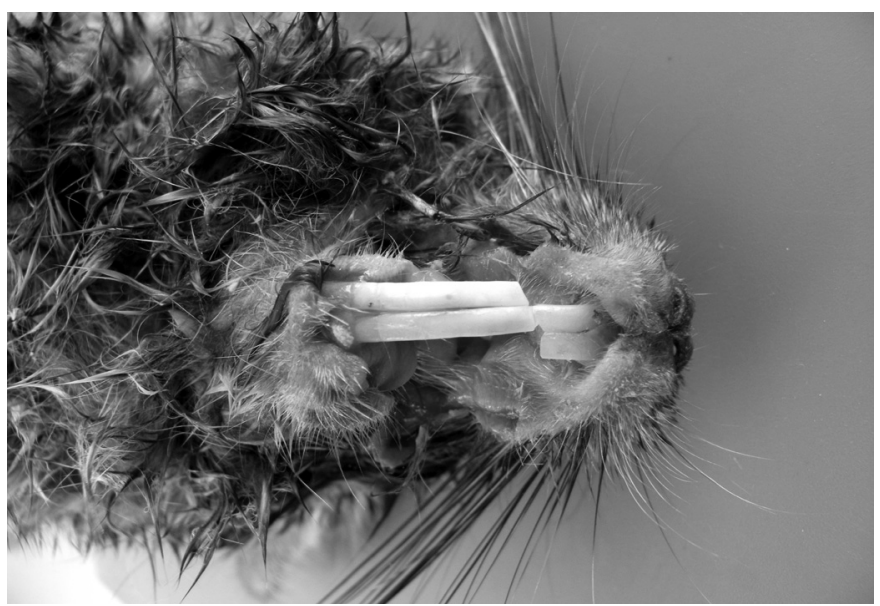

Fig.5. Desenvolvimento exagerado dos dentes incisivos de uma chinchila com grave doença dentária. Note que as coroas clínicas alongadas impedem o fechamento da boca.

\section{DISCUSSÃO}

Considerando todas as categorias de doenças, a categoria "doenças inflamatórias" apresentou o somatório de maior número de casos (52 de 202), semelhante aos estudos de necropsia realizados na década de 1950 nos Estados Unidos (Brenon 1953, Brenon 1955) e na Inglaterra (Bowden 1959). Dentre as lesões inflamatórias, as de origem infecciosa foram as mais prevalentes, principalmente as causadas por bactérias. Grande número de chinchilas tinham gastrite moderada a acentuada como principal lesão. A maioria desses casos foi possivel observar bacterias espiraladas na mucosa gástrica, que marcaram positivamente na coloração de Warthin-Starry e imuno-histoquímica com anticorpo anti-Helicobacter pylori (dados não publicados). Em uma chinchila acometida por adenocarcinoma gástrico, exame de PCR e sequenciamento genético de amostra obtida da mucosa do estômago revelou a presença de $\mathrm{He}$ licobacter com $99 \%$ de compatibilidade com Helicobacter pylori (Lucena et al. 2012a). Desde a descoberta de H. pilory como indutor de gastrite em humanos no ano de 1983 
(Warren \& Marshall 1983), este microrganismo tem sido implicado na carcinogenese gástrica baseado em diversos estudos epidemiológicos (Parsonnet 1991). Estudo experimental em gerbil da Mongólia revelou que $H$. pylori induz gastrite, metaplasia intestinal, como também adenocarcinoma gástrico em $37 \%$ dos animais infectados, comprovando a participação desta bactéria no desenvolvimento de câncer gástrico (Watanabe 1998).

Listeriose é outra doença bacteriana importante em chinchilas, descrita na forma de surtos em diferentes partes do mundo (Finley \& Long 1977, Wilkerson et al. 1997, Sabočanec et al. 2000, Kirinus et al. 2010). As chinchilas parecem ser uma das espécies animais mais sensíveis à infecção por Listeria monocytogenes, quando comparadas a outras espécies (Finley \& Long 1977). A forma encefálica de listeriose tem sido relatada (Bowden 1959), mas a maioria dos surtos descreve a doença na forma de septicemia, com predominante envolvimento hepático (Finley \& Long 1977, Wilkerson et al. 1997, Sabočanec et al. 2000, Kirinus et al. 2010). No presente estudo foram necropsiadas cinco chinchilas com a forma septicêmica da doença. Elas eram provenientes de três surtos com morbidade que variou de 6-16\% e letalidade de $100 \%$. Três desses casos haviam sido relatados anteriormente (Kirinus et al. 2010). Acredita-se que surtos de listeriose estejam relacionados ao fornecimento de ração infectada por L. monocytogenes carreada por outros roedores de vida livre, principalmente os ratos (Wilkerson et al. 1997, Sabočanec et al. 2000).

Outras chinchilas apresentaram infecção bacteriana secundária, principalmente na forma de septicemia. Infecções oportunistas causadas pela flora bacteriana normal têm sido observadas causando morte de chinchilas, seja por infecção localizada de um órgão ou como septicemia. Exemplos de bactérias oportunistas são Streptococcus sp., Staphylococcus sp., Pseudomonas sp. ou Escherichia coli. Os animais afetados geralmente estão imunocomprometidos pela idade, estado nutricional, ou estresse da criação (altas temperaturas, troca de ração etc.) (Donnelly 2004). Proteus spp. é outra bactéria que tem sido associada a casos de pneumonia, enterite, metrite e pielonefrite, por vezes em associação com outras bactérias (Bowden 1959, Donnelly 2004). Neste estudo Proteus spp. foi isolado em chinchilas acometidas por pneumonia, pileonefrite e enterite. Acredita-se que espécies de Proteus atuam como bactérias oportunistas nos tecidos já comprometidos por outros agentes ou nos casos de baixa imunidade do organismo animal (Greene 2006). As chinchilas acometidas por septicemia geralmente têm histórico de doença com evolução aguda, observado neste estudo e também no estudo das doenças de chinchilas desenvolvido na Inglaterra (Bowden 1959). Em surtos de chinchilas acometidas por septicemia deve-se procurar solucionar erros de manejo, controlar a temperatura na faixa de conforto térmico no interior do criatório, ou sanar qualquer outro fator que induza estresse nas chinchilas.

Surto de infecção por Clostridium perfringens tipo B acometeu cinco chinchilas em um lote de 395 . As lesões consistiam de extensa enterite necrosante associada a numerosos agregados bacterianos na superfície epitelial com invasão da lâmina própria, moderado edema pulmonar e degeneração gordurosa do fígado. A genotipificação de C. perfringens isolado, realizada por reação em cadeia de polimerase multiplex (mPCR), revelou C. perfringens tipo B pela detecção das toxinas alfa, beta e épsilon. Os achados sugerem $C$. perfringens tipo B como importante causador de morte súbita ou aguda em chinchilas (Lucena et al. 2011).

Intoxicação por salinomicina foi a doença isolada mais prevalente no presente estudo. No entanto, a grande representação dessa doença deve-se ao grande número de necropsias realizadas em quatro importantes surtos de intoxicação por este antibiótico ionóforo em diferentes criatórios de chinchilas no Rio Grande do Sul, após administração de ração de um mesmo lote contaminado. Foi comprovado que chinchilas são sensíveis a doses relativamente baixas de salinomicina (37 ppm), e desenvolvem grave lesão nos músculos esqueléticos (Lucena et al. 2012b).

Degeneração gordurosa do fígado predominou entre todas as lesões descritas nos laudos de necropsia. Em 16 casos esta era a lesão predominante ou única lesão observada. Anteriormente, lipidose hepática havia sido descrita como altamente sugestiva de aflatoxicose aguda, observado em uma mortandade de chinchilas na Argentina após ingestão de ração contendo 208ppm de aflatoxinas (González 2008). Porém, em estudo experimental as chinchilas demonstraram ser resistentes a aflatoxinas na dose de 200 ppm por até oito semanas de ingestão (Cepeda et al. 2011). Na nossa experiência, degeneração gordurosa do fígado manifesta-se como uma lesão secundária decorrente da diminuição da ingestão de ração em chinchilas em boa condição corporal ou obesas, seja por qualquer problema de saúde que induza inanição, ou até mesmo nas chinchilas saudáveis mantidas em jejum pré-abate.

Assim como descrito na literatura, doença dentária manifestou-se como uma importante causa de morte em chinchilas (Crossley 2001, Donnelly 2004). Má oclusão resultou do crescimento excessivo da coroa clínica dos dentes incisivos e molares e pré-molares. Como consequencia, as chinchilas desenvolveram emagrecimento e caquecia pela dificuldade de apreensão dos alimentos. 0 crescimento excessivo da porção apical do dentes maxilares resultou em compressão do canal lacrimal, manifestado por epífora e até casos de conjuntivite bacteriana secundária. A falta de atrito dentário é o principal fator implicado no alongamento exagerado dos dentes de roedores mantidos em cativeiro (Weinreb 1967), fatores adicionais como deficiência de vitamina A, também tem sido sugeridos (Wolbach 1933). Em chinchilas, acredita-se que altos níveis de cortisol no soro sanguíneo também pode predispor o crecimento dentário excessivo (Crossley 2001).

Exposição a altas temperaturas, especialmente em ambientes com alta umidade, pode resultar em hipertermia e considerável mortandade em chinchilas. A zona de conforto está entre $18,3^{\circ} \mathrm{C}$ e $26,7^{\circ} \mathrm{C}$; quando a temperatura ambiente ultrapassa muito o limiar superior os animais entram em decúbito e apresentam respiração acelerada; mucosas vermelhas que passam à cianóticas; hipersalivação viscosa e, às vezes, diarreia com sangue. A temperatura retal usualmente ultrapassa os $39,4^{\circ} \mathrm{C}$ (Donnelly 2004). Esses sinais 
clínicos foram semelhantes aos observados nas chinchilas do presente estudo. Foram acometidas principalmente chinchilas jovens e todos os casos ocorreram basicamente nos meses de verão, janeiro e fevereiro, que apresentam temperaturas mais altas. Em muitos casos houve falha no resfriamento dos galpões. As lesões macroscópicas e histológicas se restringiam a lipidose hepática leve a moderada.

Infestação parasitária limitou-se a presença de ectoparasitas e um caso de giardíase. Os casos de infestação por piolho ocorreram em chinchilas criadas como pet (uma chinchila adulta e seus dois filhotes). A infestação por pulgas ocorreu em chinchilas que tinham contato com ratos. Não são encontrados na literatura relatores de infestação por estes ectoparasitas em chinchilas. Espécies de Giardia são normalmente encontradas no intestino de chinchilas (Bowden 1959, Donelly 2004). Estresse e más condições do ambiente estão relacionados a aumento do número do parasita, resultando em diarreia e morte. 0 diagnóstico de giardíase deve ser considerado quando há quadro de diarreia associado à presença do protozoário em grande número, e exclusão de outro agente causador (Donnelly 2004).

Mortes relacionadas a agentes físicos foram mais prevalentes em animais recém-nascidos que sofreram mutilação por suas mães após o nascimento. Casos de automutilação foram vistos em chinchilas medicadas com $0,15 \mathrm{ml}$ de enrofloxacina por via intramuscular. Os animais inicialmente mutilavam as extremidades dos dedos e porção dorsal dos pés, que evoluía para completa destruição da porção distal do membro. Enrofloxacina é um antibiótico do grupo das quinolonas. Reações adversas ao uso das quinolonas têm sido descritas em diferentes espécies e estão relacionadas principalmente aos efeitos neurotóxicos da droga. Reações neurotóxicas à fluoroquinolonas descritas em humanos tratados com altas doses são numerosas e incluem dor de cabeça, tonturas, cansaço ou sonolência, visão anormal, agitação e pesadelos (Lipsky \& Baker 1999, Stahlmann \& Lode 1999). Em equinos adultos, altas doses de enrofloxacina por via intravenosa induz reações adversas como contração do focinho, excitabilidade, e comportamento apreensivo (Bertone et al. 2000). É possível que o uso de altas doses de enrofloxacina tenha induzido automutilação nas chinchilas, já que apenas os animais do criatório que receberam a injeção apresentaram o problema.

Dentre os distúrbios do trato gastrointestinal de chinchilas, casos de constipação estão os mais prevalentes (Donnelly 2004). Obstrução intestinal tem sido descrito em chinchilas mantidas à base de dietas ricas em proteínas e energia, associado à reduzida ingestão de fibras (Cousens 1963). Semelhante ao observado no presente estudo, em que os casos de constipação foram observados em chinchilas alimentadas basicamente com frutas e verduras, e baixo teor de fibras. As fezes estavam diminuídas de tamanho, ressecadas e acumuladas principalmente na porção final do cólon e reto.

Duas chinchilas apresentaram metaplasia de células adiposas no córtex da adrenal. Tinham histórico de serem agressivas. Demonstravam áreas de alopecia e nessas áreas a pele era fina e com acúmulo de gordura no subcutâneo. Hiperplasia das glândulas adrenais foi um achado inciden- tal comum em $70 \%$ das chinchilas adultas e idosas que morreram por diferentes causas. Geralmente acompanhado por hipotricose simétrica na região lateral do abdômen e dorso. Metaplasia de células adiposas do córtex adrenal foi observado em 5\% de 497 necropsias sistemáticas em humanos. Os pacientes acometidos por essa lesão tinham maior prevalência de histórico de hipertensão arterial e doença coronariana quanto comparados ao grupo controle (Saeger \& Reinhard 1998). Um estudo de 11 chinchilas relativamente saudáveis, mas com hipotricose bilateral (fur-chewing), demonstrou hiperplasia das adrenais em todos os animais. Os autores sugeriram estresse como uma possível causa para hiperplasia adrenal em chinchilas (Tisljar et al. 2002).

Não há descrição de malformações em chinchilas. Apenas um caso de malformação foi observado no presente estudo. Tratava-se de uma chinchila recém-nascida com ausência do ânus. Durante a necropsia foi constatado que também não estava presente todo o trato reprodutor, parte do cólon, o reto e a bexiga. Os rins eram policísticos. Esse tipo de anomalia é denominado atresia ani, um defeito relativamente comum em bovinos e ovinos, e que geralmente acompanha malformações urogenitais (Gilmore \& Fechheimer 1969, Dennis 1972).

\section{CONCLUSÕES}

As doenças mais comuns de chinchilas incluem doenças dentárias, gastrite, ocasionada por infecção por bactérias do gênero Helicobacter, e outras infecções bacterianas localizadas ou septicêmicas.

Lipidose hepática é uma lesão frequentemente observada na necropsia de chinchilas e sua ocorrência não está associada diretamente a nenhuma entidade específica, possivelmente está relacionada a condições que induzam a parada brusca na ingestão de ração.

A espécie é sensível a doses relativamente baixas de antibióticos ionóforos, manifestada por grave necrose muscular.

Outros distúrbios comuns incluem hipertermia devido a falhas no arejamento do ambiente nos meses de verão e lesões de origem traumática principalmente em filhotes.

Como prevenção para a ocorrência das principais doenças, as chinchilas devem ser mantidas em ambientes na zona de conforto $\left(18,3-26,7^{\circ} \mathrm{C}\right)$, alimentadas com dieta rica em fibras, e livres de fatores que possam induzir estresse, já que boa parte dos distúrbios que acomete a espécie parece ter relação direta com erros de manejo.

AGRADECIMENTOS.- Parte deste estudo foi desenvolvido e custeado pelo Programa de Cooperação Acadêmica Novas Fronteiras (PROCAD-NF) da CAPES, como também, os custos da presente publicação.

\section{REFERÊNCIAS}

Bidlingmaier T.C. 1937. Notes on genus Chinchilla. J. Mammol. 18:159-163.

Boone A.R. 1933. Three American chinchilla farms produce most costly fur. Popular Science Monthly, December 1933.

Brenon H.C. 1953. Postmortem examinations of chinchillas. J. Am. Vet. Med. Assoc. 123:310.

Brenon H.C. 1955. Postmortem examinations of chinchillas. J. Am. Vet. Med. Assoc. 126:225-226. 
Cepeda A.S., Hansen Q.A., Murcia G. \& Diaz G.J. 2011. Aflatoxicosis en chinchillas (Chinchilla lanigera). Revta Med. Vet. Zootec. Córdoba 16:23562363.

Cousens P.J. 1963. The chinchilla in veterinary practice. J. Small Anim. Pract. 4:199-205.

Crossley D.A. 2001. Dental disease in chinchillas in the UK. J. Small Anim. Pract. 42:12-19.

Dennis S.M. 1972. Atresia ani in sheep. Vet. Rec. 91:219-22.

Donnelly T.M. 2004. Disease Problems of Chinchillas. p.254-265. In: Quesenberry K.E. \& Carpenter J.W. (Eds), Ferrets, Rabbits, and Rodents: Clinical Medicine and Surgery. $2^{\text {nd }}$ ed. Saunders Elsevier, St Louis. 261p.

Finley G.G. \& Long J.R. 1977. An epizootic of listeriosis in chinchillas. Can. Vet. J. 6:164-167.

Gilmore L.O. \& Fechheimer N.S. 1969. Congenital abnormalities in cattle and their general etiologic factors. J. Dairy Sci. 52:1831.

González P.M.L., Carvalho E.C.Q., Tissera J.L., Keller K.M., Magnoli C.E., Rosa C.A.R., Dalcero A.M. \& Cavaglieri L.R. 2008. An outbreak of acute aflatoxicosis on chinchilla (Chinchilla lanigera) farm in Argentina. J. Vet. Diagn. Invest. 20:853-856.

Greene C.E. 2006. Infectious Diseases of the Dog and Cat. $3^{\text {rd }}$ ed. Saunders Elsevier, St Louis. 1387p.

Jiminez J.E. 1996. The extirpation and current status of wild chinchillas Chinchilla lanigera and C. brevicaudata. Biol. Cons. 77:1-6.

Kirinus J.K., Krewer C., Zeni D., Monego F., Silva M.C., Kommers G.D. \& Vargas A.C. 2010. Surto de listeriose sistêmica em chinchillas. Cien. Rural. 40:686-689.

Lipsky B.A. \& Baker C.A. 1999. Fluoroquinolone toxicity profiles: A review focusing on newer agents. Clin. Infect. Dis. 28:352-364.

Lucena R.B., Farias L., Libardoni F., Vargas A.C., Giaretta P. \& Barros C.S.L. 2011. Necrotizing enteritis associated with Clostridium perfringens Type B in chinchillas (Chinchilla lanigera). Pesq. Vet. Bras. 31:10711074.

Lucena R.B., Rissi D.R., Queiroz D.M.M. \& Barros C.S.L. 2012a. Infiltrative gastric adenocarcinoma in a chinchilla (Chinchilla lanigera) naturally infected by Helicobacter pylori. J. Vet. Diagn. Invest. (Em publicação)

Lucena R.B., Fighera R.A., Tessele B., Giaretta P.R. \& Barros C.S.L. 2012b. Surtos de intoxicação por salinomicina em chinchilas (Chinchilla lanige$r a$ ). Pesq. Vet. Bras. 32(1):43-48.

Parsonnet J., Vandersteen D., Goates J., Sibley R.K., Pritikin J. \& Chang Y. 1991. Helicobacter pylori infection in intestinal- and diffuse-type gastric adenocarcinomas. J. Natl Cancer Inst. 83:640-643.

Sabočanec R., Čuljak K., Ramada K., Naglić T., Šeol B. \& Matičić D. 2000. Incidence of listeriosis in farm chinchillas (Chinchilla laniger) in Croatia. Vet. Arch. 70:159-167.

Saeger W. \& Reinhard K. 1998. Fat-Cell metaplasia in the adrenal cortex: incidence, structure, and correlation to basic diseases in a postmortem series. Endocr. Pathol. 3:241-247.

Stahlmann R. \& Lode H. 1999. Toxicity of quinolones. Drugs 58(Suppl.2):37-42.

Tisljar M., Janić D., Grabarević Z., Simpraga B., Marinculić A., Pinter L., Janicki Z. \& Nemanic A. 2002. Stress-induced Cushing's syndrome in furchewing chinchillas. Acta Vet. Hung. 50:133-42.

The Eurogast Study Group 1993. An international association between Helicobacter pylori infection and gastric cancer. Lancet 341:13591362.

Warren J.R. \& Marshall B.J. 1983. Unidentified curved bacilli on gastric epithelium in active chronic gastritis. Lancet 1:1273-1275.

Watanabe W., Tada M., Nagai H., Sasaki S. \& Masafumi N. 1998. Helicobacter pylori induces gastric cancer in Mongolian gerbils. Gastroenterol. 115:642-648.

Weinreb M.M., Assif D. \& Michaeli Y. 1967. Role of attrition in the physiology of the rat incisor. I The relative value of different components of attrition and their effect upon eruption. J. Dent. Res. 46:527-531.

Wilkerson M.J., Melendy A. \& Stauber E. 1997. An outbreak of listeriosis in a breeding colony of chinchillas. J. Vet. Diag. Invest. 3:320-323.

Wolbach S.B. \& Howep R. 1933. The incisor teeth of albino rats and guinea pigs in vitamin A deficiency and repair. Am. J. Pathol. 9:275-293. 